\section{Save That Dye!}

Michael Titford, University of South Alabama MTitford@usouthal.edu

All laboratory workers face the ongoing requirement that all chemicals and solutions used in the lab be labeled with the date of receipt or preparation, together with the expiration date of the chemical and the initials of the preparer, as well as any known hazards associated with that chemical. Officials from accrediting agencies, sometimes unannounced, pick and poke around laboratories looking for outdated chemicals and solutions, including biological stains left unsuspectingly at the back of a fridge or on a seldom used shelf. If they find such an outdated stain or chemical a citation can result that must be addressed before further accreditation is given. This has resulted in a knee jerk reaction in many laboratories, with a good many dyes and stains discarded before their time. (For the purposes of this article, dyes refer to the dry powders and stains refer to staining solutions, ready to use.) Surprisingly, the dyes kept on hand in histology for the preparation of staining solutions, for the most part, have no expiration date and can be kept for long periods. However, that is not to say that all dyes keep for ever, or that unstable dyes can be kept for long periods. And most certainly a dye that did not work when first purchased will not improve with age!

To appreciate the longevity of histological dyes, some knowledge of the history of dye manufacture is needed. The dye industry was created in 1856 in London when William Perkin created the color mauve from aniline while attempting to synthesize quinine. Aniline was available in large quantities from coal tar, a by-product of the recently created coal gas industry. Following Perkin's discovery, dye industries sprung up around Western
Europe, usually in industrial areas close to the site of the coal gas industry and close to a river. Companies we now associate with pharmaceuticals such as Ciba, Giegy, Hochst, Agfa and Bayer were prominent or even founded during the early period of the dye industry.

The major use of dyes then, as now, was in staining of fabrics. Large sums of money could be made by a manufacturer of a new color dye that was "fast" i.e., that bound well to the fabric and resisted fading from the sun or repeated washings. Many of the techniques familiar to histologists were first used to bind dyes to fabrics. Pre and post Mordanting, auxiliary chemicals and heat application to enhance staining were first used in the dye industryt.

Competition was fierce. When a new dye was patented, others in the field would prepare the dye and study its structure, then prepare the dye by a different chemical method thus avoiding patent infringement. Or perhaps a slightly different hue was obtained by slight changes in the molecular structure. Other differences were created during manufacture. The solid dyes were "salted" out of solution by different methods and other chemicals were added to the dyes to stabilize the staining of fabrics. Rather confusing to us now is the names used for dyes and the use of synonyms. Often the dye was named after a place or a flower of a plant whose color the dye resembled. However, if the dye was made by another company under patent, or by a slightly different method, the dye might be given another name. Lillie ${ }^{2}$ reports that some dyes can have up to 40 synonyms. And then, some dyes were just named incorrectly!

Biologists of the time such as Ehrlich, Schmorl and Unna searched through the hundreds of newly available dyes looking for those that had applications in the staining of biological tissues.

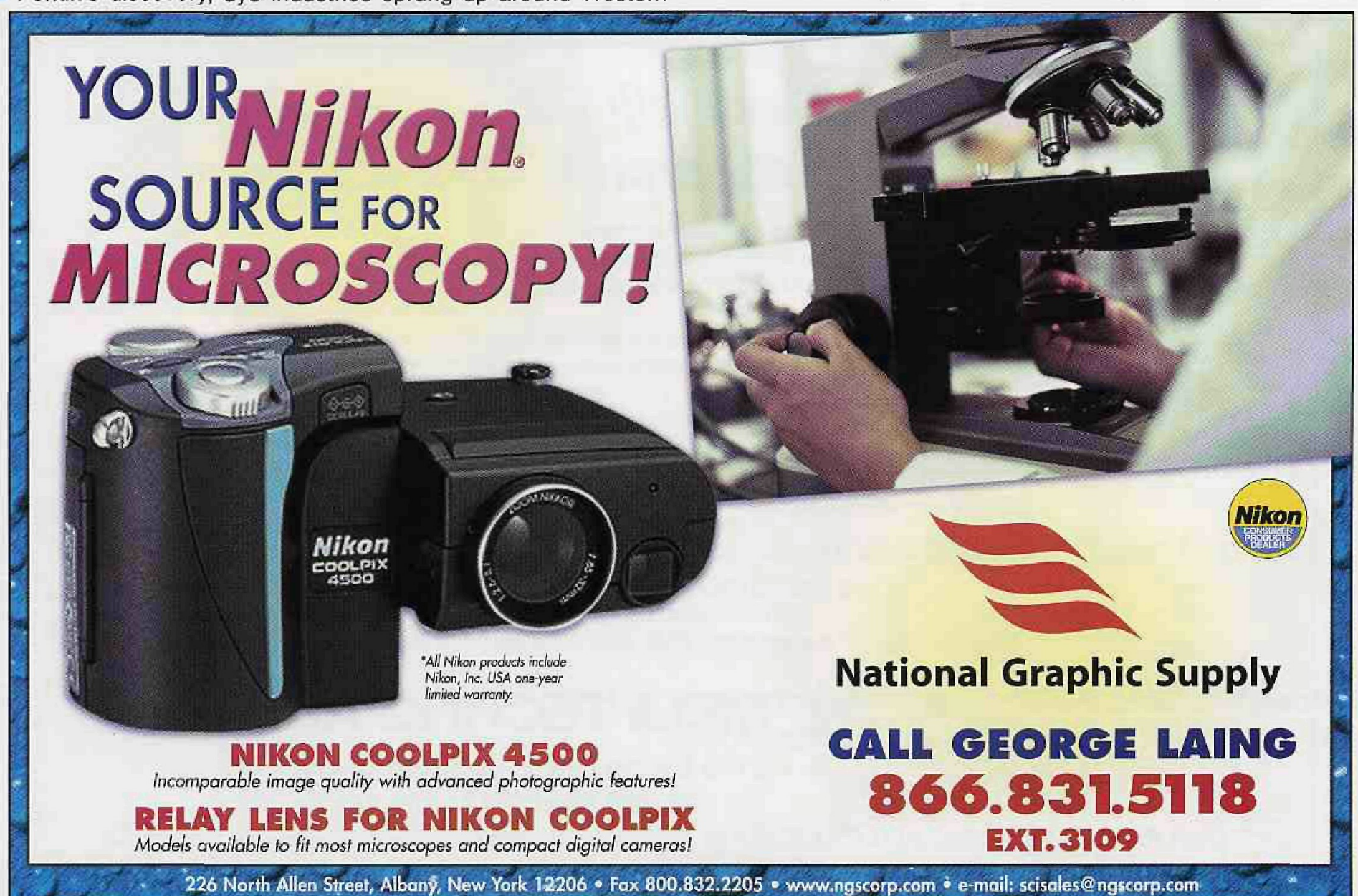

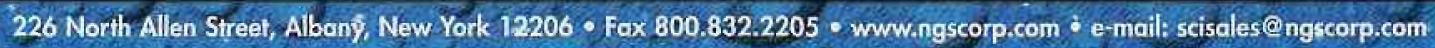


Soon companies sprung up supplying these new dyes to biologists. The most famous of these was the Grübler Company based in Leipzig, Germany. George Grübler the founder obtained samples of dyes from the new industry, and using secret empirical methods tested them on biological tissues for their use in science. Those he found acceptable he purchased in bulk, repackaged, and sold to biologists. Soon Grübler dyes developed a reputation as the best dyes for use in biological studies. Prominent scientists of that time recommended Grübler dyes in their writings. Van Gieson for example recommended Grübler dyes, as did Mallory ${ }^{3}$.

World Wars I and II created an embargo on German dyes and spurred the development of the dye industry in the United States. The first dyes manufactured in America were often inferior to the German dyes and concerned scientists created the Biological Stain Commission to test and certify biological stains for use. In more recent years the Society of Dyers and Colourists in Bradford, England have created the color index number system based on the dyes chemical structure. Between them, these two organizations have assisted histologists by standardizing the dyes used to stain biological tissues.

A few years ago this writer was given a box of old dyes, mainly Grübler with a few Aniline Dye Company samples that had been purchased at an auction of medical memorabilia. More recently other Grübler and National Aniline \& Chemical Company samples have been donated. Some of these dyes had never been opened. Others were in partially filled bottles. The Grübler dyes were prepared between 1880 and 1914. A study was undertaken to compare these dyes with modern day equivalents. Fourteen were selected for the study. These dyes were studied using spectrophotometry and in a variety of histological special stains

\section{Psst. Buddy ...}

Need a Fluorescence Reference?

\section{Fluor-Ref ${ }^{\text {Tm }}$}

Fluorescence Reference Slides

Great for Lamp centration, testing power settings, confirming staining consistency and standardizing confocal settings.

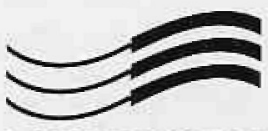
MICROSCOPYI

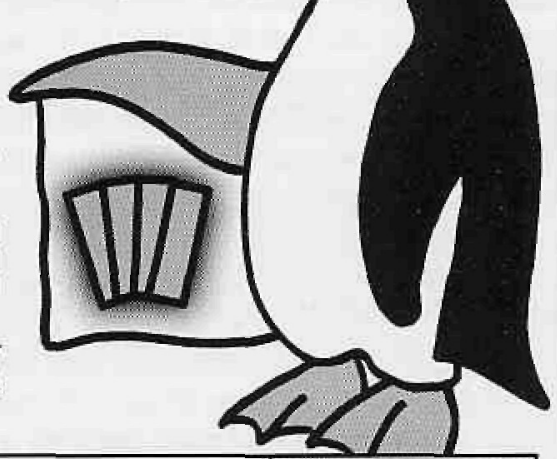

\begin{tabular}{|l|r|}
\hline Single slides & $\$ 18$ \\
\hline Set of 4 (R, G, B/UV, Orange) & $\$ 55$ \\
\hline Classroom sets: 6 sets of 4 & $\$ 275$ \\
\hline
\end{tabular}

To Order: 413-746-6931 or www.MicroscopyEducation.com

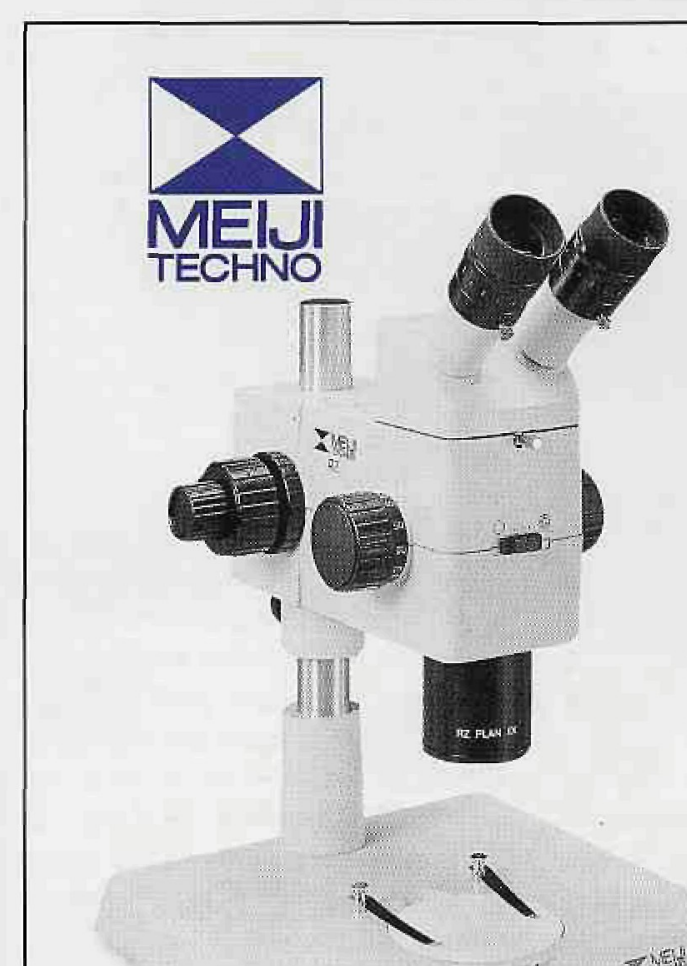

\section{The Meiji RZ Series of Research Stereo Microscopes.}

If you are looking for precision, durability, quality and value in a high performance Stereo Microscope, we invite you to take a closer look at Meiji's RZ Series of Research Stereo Microscopes.

The $R Z$ Series modular system design allows you the freedom to create an ideal instrument for your specific need or application. Featuring a 10:1 zoom ratio, variable double iris diaphragm, and positive detente click stops at 12 positions of magnification. A full range of optional accessories is available, including: Video and photo-micrographic systems, brightfield-darkfield transmitted light stands, ergonomic binocular head, drawing attachment, multiple interchangeable objectives and wide-field eyepieces. Complete system versatility backed by a "Limited Lifetime Warranty."

For more information on these economically priced Stereo Microscopes, please call, FAX, write us or log on to our website today.

\section{MEIJI TECHNO AMERICA}

2186 Bering Drive, San Jose, CA 95131 , Tel: 408.428 .9654 , FAX: 408.428 .0472

Toll Free Telephone: 800.832 .0060 or visit our website at www.meijitechno.com 
in common use today. Where possible, modern day dyes were used as controls.

The diamond fuchsin and fuchsin-bacillus dyes were both samples of basic fuchsin. Both samples had a maximum absorbance of $548 \mathrm{~nm}$. They both stained well in the Ziehl Neelsen method for acid fast bacteria. When both were used to prepare Schiff's reagent for the PAS reaction, the results were inferior to modern day samples of basic fuchsin.

A Sudan III sample had a maximum absorbance of $509 \mathrm{~nm}$ in alcohol and stained lipid material in frozen sections of adrenal gland, but a paler shade of red than the modern day oil red $O$ used as a control. Scarlet $R$ is one of the names that has had different uses in the industrial dye industry. This sample dissolved in organic solvents and was originally thought to be Sudan IV. It dissolved in organic solvents and gave only a pale, off red coloration to lipids in frozen sections. Its maximum absorbance in alcohol was $513 \mathrm{~nm}$. An orange $\mathrm{G}$ sample had an identical spectral curve to the modern day control $(479 \mathrm{~nm})$ and gave excellent results in the Mallory trichrome stain and the PAS-hematoxylinorange $\mathrm{G}$ method for acidophil cells in the anterior pituitary. An aniline blue had a spectral absorbance of $595 \mathrm{~nm}$ and gave excellent results in the Mallory trichrome method.

The Pyronin sample had a maximum absorbance of 549 $\mathrm{nm}$ and spectral curve that most closely resembled that of pyronin $Y$. When used in the methyl green-pyronin $Y$ method for RNA and DNA, this sample gave the best results with Carnoy-fixed material. The fuchsin $S$ acid and rubin $S$ were both examples of acid fuchsin and had close maximum spectral absorbencies (fuchsin S 546 $\mathrm{nm}$, rubin S $550 \mathrm{~nm}$ ) and similar spectral curves with the fuchsin $\mathrm{S}$ sample having a shoulder at $500 \mathrm{~nm}$. Lillie ${ }^{2}$ discusses this dye

Scalpels to Scoops to Screwdrivers to Spatulas to Speedles to Sputter Coaters Carbon Coaters to Clip Mounts to Carbon Rods to Custom Equipment Beakers to Beam Stops to Boats to Books to Bottles to Boxes Tape to Timers to Titanium Tweezers to Tensile Testers Pipettes to Planchets to Pithwood to Power Supplies Hacksaws to Hex Grids to Heating Stages Universal Holders to Uranyl Acetate: Vacuum Pumps to Viewing Boxes Wafer Tweezers to Work Holders Magnifiers to Micromanipulators Desiccators to Dropping Bottles Latex Spheres to Lens Tissue EFFA Dusters to Evaporators Glass Bottles to Grid Boxes Razor Blades to Ruby Mica Ferritin to Films to Forceps Acetone to Aperturés an al What can you imagine?

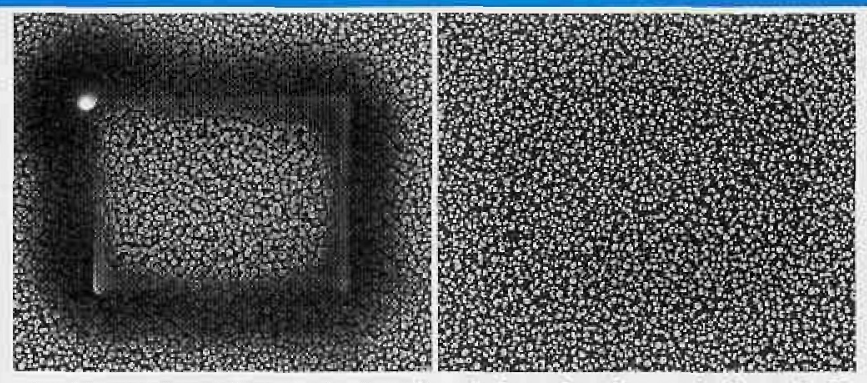

(4) res
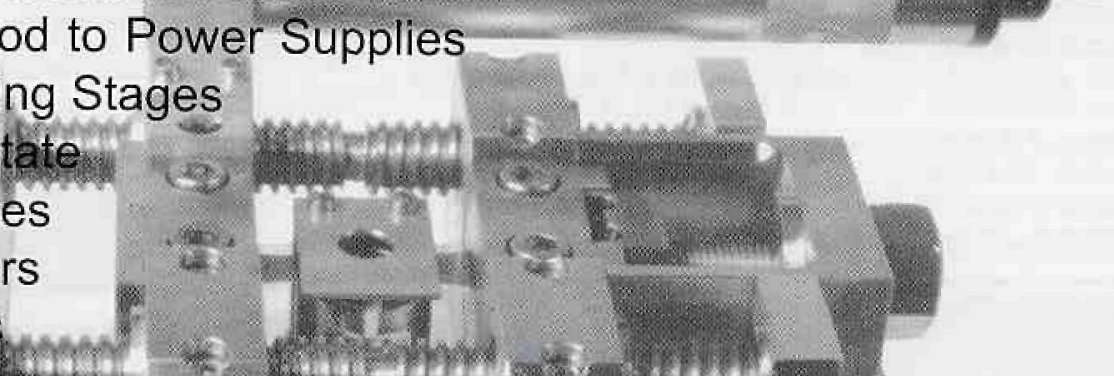

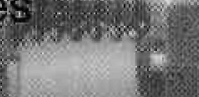
ERNEST F. FULLAM, INC.

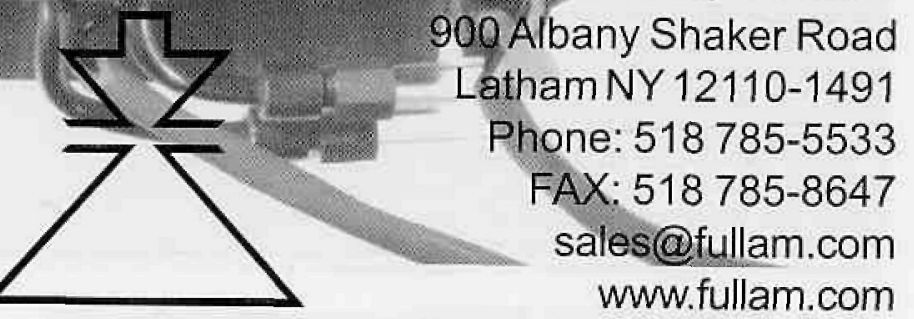


in detail and states that the dye usually contains varying amounts of rosaniline, pararosaniline, magenta II and new fuchsin. These samples probably contained different amounts of these constituents. A safranin $O$ water soluble and safranin yellow water soluble samples both had a maximum absorbance at $532 \mathrm{~nm}$ and identical spectra. Both gave adequate results in the Gram stain (Lillie) and stained umbilical cord matachromatically. A Grübler carmine sample had the typical bright red carmine color and was the natural dye obtained from the insect, Coccus cacti. This dye gave adequate results with the best carmine method for glycogen and the lithium carmine method for nuclei. Aurantia is an obsolete textile dye related to picric acid. Lillie $^{2}$ describes it as poisonous and explosive. When used in place of the picric acid in the Van Gieson method, connective tissues stained bright red orange with yellow cytoplasm. Its absorbance was $415 \mathrm{~nm}$ in alcohol.

Hematein is a naturally occurring dye extracted from the logwood tree, Haematoxylon campecianum. It is the oxidized form of hematoxylin. This sample gave poor results with the Mallory phosphotungstic acid hematoxylin and Heidenhain iron hematoxylin methods compared to the modern day control. A methyl eosin was assumed to be a member of the eosin group of dyes of which the alcohol soluble eosin $Y$ (C.I. 45380) is most popular today. This sample dissolved poorly in water and gave bland results when used in a routine hematoxylin and eosin method. Only strongly acidophilic tissues stained with the dye. The graduated shades of pink associated with good eosin staining were missing.

Conclusion

Biological dyes can retain their staining characteristics up to one hundred years after manufacture, if kept sealed and in a cool, dry atmosphere. There are some caveats to this statement. Stains that did not work well immediately after manufacture will certainly not work well after long storage. Also dyes or mixtures of dyes and other chemicals in which there are ongoing chemical reactions such as oxidation may well become inactivated after time. The Romanowsky type dyes such as Giemsa, Leishman and Wright also involve mixtures of dyes and chemicals where optimum reaction time may have passed by.

However, for all old biological dyes, histologists are urged to try them out using good control material and see their appearance in stained control tissue sections before making a decision to throw them out ${ }^{4}$. The development of the dye manufacturing industry is an interesting subject for the histologist interested in history ${ }^{1}$.

\section{References}

1) Beer, J. J. 1959. The Emergence of the German Dye Industry. University of Illinois Press

2) Lillie, R.D. 1977. H.J. Conn's Biological Stains. Williams and

Wilkins, Baltimore

3) Mallory, F.B. and J.H. Wright 1901. Pathological Technique. WB Saunders. New York.

4) Titford, M. 2001. Comparison of historic Grübler dyes with modern counterparts. Biotech \& Histochem. 76:23 - 30.

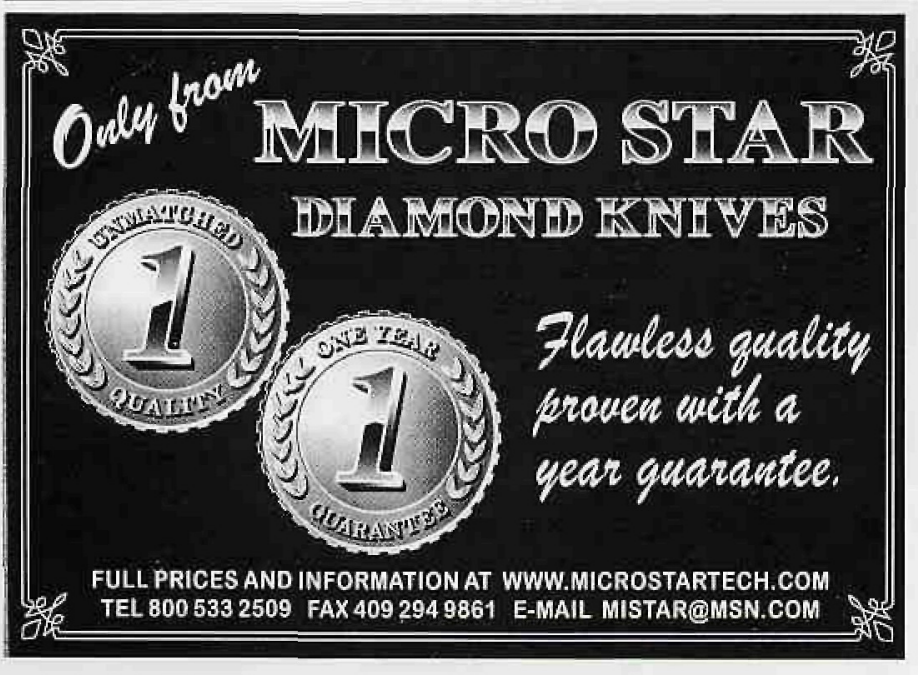

\section{IT'S SHOW TIME!}

Watch Your SEM Perform with Quality Scintillator Light Guides From

\section{E. Taylor Engineering, Inc.}

- Light Guides for Leo/Cambridge model SEMs - $\$ 495$

- Custom Scintillators for other model SEMs - $\$ 89$

- P47 Phosphor Coating

- Highest Quality Engineering

- See our web site for special offers on maintenance and re-coating specials

M. E. Taylor Engineering, Inc. 21604 Gentry Lane

Brookeville, MD 20833

Phone: (301) 774-6246

\section{See us on the Web @} www.semsupplies.com

\section{USEP EOUIPMEMT FOR SAL}

Zeiss EM 902 in excellent condition.

Call: Horst Harry Welbat.

Zeiss fatory trained TEM preventative maintenance specialist

(Independant)

(805) 531-0009

\section{POSITIOI DESIREP}

Microscopist / Materials Scientist seeking a challenging career opportunity. Fourteen years of industrial light microscopy, SEM/EDS, XRD, Micro-FTIR, XRF, image analysis, and particle sizing experience. Interested parties please contact Lou Sobello at njlou1960@juno.com or (609) 265-0433 for resume and references.

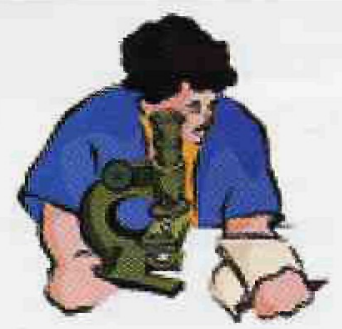


NIKON NAMES HIRO KUSAKA AS PRESIDENT \& CEO OF NIKON INSTRUMENTS, INC.

Nikon Instruments, Inc. announced today that Hiro Kusaka has been appointed as its President and CEO. Mr. Kusaka succeeds Takeshi Hirai, who was recently named President of Nikon Geotecs Co. Ltd in Japan. In his new role in Melville, New York, Mr. Kusaka will be responsible for sales, customer service, and new product development, as well as to further expand Nikon's presence in the digital applications business in the science and industrial markets.

\section{Image analySIS magazine}

The latest issue of Soft Imaging System's magazine with life sciences as its main focus is available now. "Life sciences" is a very broadly interpreted term referring to all branches of research in the natural sciences that investigate living organisms and how they function, ranging from the macroscopic to the sub-molecular level. The conventional delineation between biology, chemistry and physics has become much less clear. And new findings are often applied very quickly. Our "Image analySIS magazine" is published several times a year in both English and German. If you would like your name added to our mailing list, simply let us know. It's free! There's also an online version you can download at Soft Imaging System's website (www.soft-imaging net). Soft Imaging System is one of the signpost suppliers for digital image analytical systems and digital cameras in the fields of light- and electron microscopy.

Nikon's DN100 Digital Network Camera Delivers Revolutionary Capabilities for Industrial and Life Science Applications

For industrial inspectors, pathologists and researchers who have longed for a simple to use digital camera with networking capabilities, the wait is over. Nikon's new DN100 digital network camera captures, transfers and monitors micro/macro images simultaneously. (Photo: http://www.newscom.com/cgi-bin/prnh/ 20010614/NYTH089) The DN100 delivers color 1.3 megapixel resolution at a rate of 15 frames per second and alleviates the need to first record images before sharing them. Captured images can be transferred to networked parties simultaneously at 1 frame per 2 seconds through 1 frame every 10 minutes, making it ideal for time lapse applications. Users also have the option of directly storing images on the PCMCIA type II card slot with a compact flash cord or memory stick using the appropriate adapter. Downloading data to an FTP server for distribution, printing and archiving is another option. For more information on the DN100 Digital Network Camera, please contact Nikon Instruments Inc. 1300 Walt Whitman Road, Melville, NY 11747; phone 800-52Nikon, ext. P1032, or visit the Nikon website and MicroscopyU at http://www.nikonusa.com.

NORAN System SIX X-Ray Microanalysis Package Introduced at Microscopyand Microanalysis 2002

Thermo NORAN, announced at Microscopy and Microanalysis 2002 , the release of its latest $X$-ray microanalysis system. Named the NORAN System SIX, this microanalysis package includes Thermo NORAN's newest high-throughput acquisition and digital beam control electronics, and a single, unified software program for all acquisition, analysis, and reporting. Design of the NORAN System SIX is a direct result of feedback and suggestions provided by existing and prospective customers.

With Spectral Imaging, users collect $X$-ray and image data sets of up to $1024 \times 1024$ pixels. The result is a full deadtime- corrected spectrum at every pixel. This data presents a complete, archival record of the sample. Users can extract spectra, X-ray maps, and linescans from the data set.

The new COMPASS automated statistical-analysis package evaluates the spectral imaging data, looking for spectral shape similarities. COMPASS sorts similar-appearing spectra into primary components, quickly generating 'pure' component maps with single-pixel sensitivity. A 2001 Macres Award winner and a 2002 R\&D 100 winner, COMPASS is exclusively licensed to Thermo NORAN by Sandia National Laboratories. Contavt: Dennis Masaki, Director of Marketing Thermo NORAN, (608) 831-6511 E-mail: dennis.masaki@thermo.com www.thermo.com/noran

\section{StockerYale Announces Mille Luce TM Model M1000 Illuminator}

The Mille Luce TM Model M1000 is a lightweight, stackable and ultra-compact illuminator. A slide potentiometer that adjusts lamp intensity and iris dimming is an available option. The innovative optical design greatly extends lamp life without a loss of light output. Other features include: quick and easy lamp change, an integrated IR filter and a whisper fan. StockerYale can be reached at 32 Hampshire Road, Salem, new Hampshire, 03079. (603) 893-8778, www.stockeryale.com.

\section{LEO SUPRA FESEM range introduced at M\&M}

LEO Electron Microscopy has introduced a new range of field emission scanning electron microscopes providing ultra high resolution imaging combined with full analytical capabilities for nanoscale applications. Semiconductor and nanoscale technology applications benefit from $20 \%$ resolution improvement in the low voltage range (now $1.7 \mathrm{~nm} @ 1 \mathrm{kV}$ and $4.0 \mathrm{~nm} 0.1 \mathrm{kV}$ ) and the variable pressure technology for investigating non-conducting specimens without prior preparation. The improved high current mode ads analytical capabilities when the need arises. For life science the improved high efficiency In-lens detector (with $250 \%$ higher sensitivity), coupled with improved resolution the SUPRA series enables imaging beyond previous limits. The large chamber with the large fully motorised specimen stages are ideally suited for cryo-applications.

\section{LEO Extended Pressure SEM}

LEO Electron Microscopy has further pushed back the frontiers of SEM design with the introduction of extended pressure SEM's which open new fields of application in the life sciences, pharmaceuticals, and materials analysis. These new microscopes; the 1450 EP and the 1455EP, provide the capability to image and analyse specimens whilst a pressure as high as $3000 \mathrm{~Pa}$ is maintained in the specimen chamber. The instruments maintain excellent performance in the both high vacuum (HV) and variable pressure (VP) modes which have developed as a characteristic of the 1400 series instruments.

\section{LEO CONCISE ${ }^{\oplus}$ graphical user interface for LEO SEMs}

LEO Electron Microscopy introduces CONCISE ${ }^{a}$, a new structured graphical user interface (GUI) for the current and future ranges of multi purpose SEMs and FESEMs.

LEO $1540 \times B$ CrossBeamB The ultimate $3 D$ research tool

The all-new LEO 1540XB CrossBeam ${ }^{6}$ workstation is the perfect tool for three dimensional analysis with no compromises on imaging capabilities. For all of these products contact: LEO Electron Microscopy Group, www.leo-em.com, (800) 356-1090. 